\title{
Sexually Dimorphic Regulation of EET Synthesis and Metabolism: Roles of Estrogen
}

\author{
An Huang* and Dong Sun \\ Department of Physiology, New York Medical College, Valhalla, NY, United States
}

Epoxyeicosatrienoic acids (EETs) are metabolites of arachidonic acid via cytochrome P450 (CYP)/epoxygenase and are hydrolyzed by soluble epoxide hydrolase (sEH). Circulating and tissue levels of EETs are controlled by CYP (EET synthesis) and sEH (EET degradation). Therefore, both increases in CYP activity and decreases in sEH expression potentiate EET bioavailability, responses that prevail in the female sex as a function of estrogen. This mini review, based on subtitles listed, briefly summarizes studies focusing specifically on (1) female-specific potentiation of CYP/epoxygenase activity to compensate for the endothelial dysfunction; and (2) estrogen-dependent downregulation of sEH expression, which yields divergent actions in both systemic and pulmonary circulation, respectively.

OPEN ACCESS

Edited by:

John D. Imig,

Medical College of Wisconsin,

United States

Reviewed by:

Mohammed A. Nayeem,

West Virginia University, United States Yi Zhu,

Tianjin Medical University, China

*Correspondence:

An Huang

an_huang@nymc.edu

Specialty section:

This article was submitted to Translational Pharmacology,

a section of the journal

Frontiers in Pharmacology

Received: 04 September 2018 Accepted: 08 October 2018

Published: 29 October 2018

Citation:

Huang A and Sun D (2018)

Sexually Dimorphic Regulation of EET

Synthesis and Metabolism:

Roles of Estrogen.

Front. Pharmacol. 9:1222.

doi: 10.3389/fphar.2018.01222

\section{Estrogen-Potentiating EET Synthesis in Response to Endothelial Dysfunction:} This section summarizes the current understanding regarding the roles of estrogen in facilitating EET synthesis in response to endothelial dysfunction. In this regard, estrogen recruitment of EET-driven signaling serves as a back-up mechanism, which compensates for $\mathrm{NO}$ deficiency to preserve endothelium-dependent vasodilator responses and maintain normal blood pressure.

Estrogen-Dependent Downregulation of Ephx2/sEH Expression: This section focuses on molecular mechanisms responsible for the female-specific downregulation of sEH expression.

Roles of EETs in Systemic Circulation, as a Function of Estrogen-Dependent Downregulation of sEH: This section summarizes studies conducted on animals that are either deficient in the Ephx2 gene (sEH-KO) or have been treated with sEH inhibitors (sEHIs), and exhibit EET-mediated cardiovascular protections in the cerebral, coronary, skeletal, and splanchnic circulations. In particular, the estrogen-inherent silencing of the Ephx2 gene duplicates the action of sEH deficiency, yielding comparable adaptations in attenuated myogenic vasoconstriction, enhanced shear stress-induced vasodilation, and improved cardiac contractility among female WT mice, male sEH-KO and sEHItreated mice.

Roles of Estrogen-Driven EET Production in Pulmonary Circulation: This section reviews epidemiological and clinical studies that provide the correlation between the 
polymorphism, or mutation of gene(s) involving estrogen metabolism and female predisposition to pulmonary hypertension, and specifically addresses an intrinsic causation between the estrogen-dependent downregulation of Ephx2 gene/sEH expression and female-susceptibility of being pulmonary hypertensive, a topic that has never been explored before. Additionally, the issue of the "estrogen paradox" in the incidence and prognosis of pulmonary hypertension is discussed.

Keywords: epoxyeicosatrienoic acids, soluble epoxide hydrolase, sex, estrogen, pulmonary hypertension

\section{INTRODUCTION}

It is established that oxidative metabolism of arachidonic acid through the cyclooxygenase (COX) and lipoxygenase pathways to biologically activate eicosanoids plays a critical role in the regulation of pathophysiological processes. To date, a dubbed "third pathway" of the cytochrome P450 (CYP)/epoxygenase system has come to the forefront of research with aims to evaluate the pathophysiological significance of its biologically active mediators, epoxyeicosatrienoic acids (EETs) (Roman, 2002). EETs possess cardiovascular protective properties in the systemic circulation via endothelium-derived hyperpolarizing factor (EDHF)-based vasodilator responses (Archer et al., 2003; Fleming, 2004; Huang et al., 2004, 2005) to lower blood pressure in both physiological (Imig, 2012; Sun et al., 2014) and pathological conditions (Lee et al., 2010; Ma et al., 2013). The contribution of EETs toward the cardiovascular protection can be controlled by soluble epoxide hydrolase (sEH), an enzyme that hydrolyzes EETs to their biologically inactive diols (dihydroxyeicosatrienoic acids, DHETs) (Newman et al., 2005). As such, either the potentiation of EET synthesis or the reduction of EET metabolism is able to increase EET bioavailability, and therefore, both CYP/epoxygenase and $\mathrm{sEH}$ can be therapeutic targets for cardiovascular diseases. More importantly, both enzymatic activities are regulated by female hormones/estrogens, leading to a sex disparity in the presentation of EET-mediated contributions. In general, beneficial actions of EETs in the cardiovascular system have been well reviewed (Roman, 2002; Imig, 2012), however, the sexually dimorphic phenotype, in terms of female-favorable contributions of EETs is much less addressed. Thus, this mini-review will summarize studies from the authors', as well as others' laboratories, focusing on (1) female-specific potentiation of CYP activity to compensate for the endothelial dysfunction; and (2) estrogen-dependent suppression of $\mathrm{sEH}$ expression that yields divergent actions in the systemic and pulmonary circulation, respectively.

\section{ESTROGEN-FAVORABLE EET SYNTHESIS IN RESPONSE TO NITRIC OXIDE DEFICIENCY}

Cytochrome P450 are encoded by a complex superfamily of genes; they are located in the endoplasmic reticulum and add an epoxide across one of the four double bonds of arachidonic acid to produce four EET regiosomers: 5,6-EET, 8,9-EET, 11,12-EET and 14,15-EET. The CYP2C and CYP2J families are responsible for the majority of EET generation in mammals (Imig, 2012). Specifically, CYP2C29 and CYP2C7 are EET synthasase in mouse and rat vascular endothelium, and express predominantly in female vessels deficient in NO synthesis (Sun et al., 2010, 2011).

One of rationales for investigating CYP/epoxygenase function is its compensatory nature, characterized by the fact that the enzymatic activity and its contribution to the regulation of cardiovascular function are dampened under physiological conditions, and become discernible in most instances, only with endothelial dysfunction, manifested as impaired NO bioavailability. Therefore, most in vitro studies aiming to evaluate CYP activities were performed in the presence of inhibitors of endothelial nitric oxide synthase (eNOS) and COX. More intriguingly, the CYP/EET-evoked compensatory action exerts in a female favorable manner, as indicated by the evidence that in eNOS and COX-1 double knockout (KO) mice, EET-mediated responses via an EDHF-based event contribute significantly to the preservation of endothelium-dependent relaxation, coinciding with normal blood pressure in female animals (Scotland et al., 2005), with little of this compensation in their male counterparts that display hypertension, associated with impaired endothelium-dependent vasodilations (Brandes et al., 2000). The same responsive pattern was also observed in the high fructose-induced metabolic syndrome or chronic insulin-loading animal models, where only hyperinsulinemic male rats, not females, developed hypertension, even though both sexes displayed endothelial dysfunction (Galipeau et al., 2002; Vasudevan et al., 2005); moreover, female ovariectomy $(\mathrm{OV})$ prevented, and $\mathrm{OV}$ with estrogen replacement (OVE) restored the normotension (Galipeau et al., 2002; Song et al., 2005). These findings clarify estrogen as an essential player in the compensation against endothelial dysfunction (deficiency of $\mathrm{NO}$ and/or PGs), via perhaps, recruiting EET/EDHF-dependent signaling.

In the microcirculation, estrogen, in response to $\mathrm{NO}$ deficiency, affords protection via unveiling the EET/EDHFmediated pathway as a back-up mechanism, to maintain normal microcirculatory resistance. For instance, in female eNOS$\mathrm{KO}$ mice and female rats treated with L-NAME, estrogen via activation of estrogen receptors (ERs), evokes a solely EETmediated response that fully preserves shear stress-induced vasodilation (SSID, one of the most important local regulators in the control of microcirculatory resistance) (Huang et al., 2001a,b; Wu et al., 2001), reminiscent of a significantly smaller magnitude of SSID mediated by COX-derived prostaglandins 
(PGs) in male eNOS-KO and L-NAME treated counterparts (Sun et al., 1999, 2006). Therefore, the female phenotype of SSID is defined as augmented vasodilator responses mediated by EETs in an EDHF-based approach, as a function of either decreased NO, or increased EET bioactivities (Huang and Kaley, 2004), highlighting further, a reverse interaction between the two endothelial mediators (NO vs. EETs). The female phenotypic SSID (EET-mediation) can be changed to male phenotype of SSID (PG mediation) when gonad-intact females are ovariectomized (Huang et al., 2001b); vice versa, in vitro exposure of male vessels to a physiological concentration of estrogen enables to elicit a female phenotype of SSID (Huang et al., 2004). Thus, in the deficiency/impairment of NO bioactivity, vascular release of EETs to maintain a normal endothelial sensitivity to shear stress is dependent of estrogen and occurs via an ER-mediated activation of a PI3K/Akt pathway to upregulate CYP2C29 and CYP2C7 genes (Huang et al., 2004; Sun et al., 2011).

\section{ESTROGEN-DEPENDENT DOWNREGULATION OF Ephx2/sEH EXPRESSION}

Mammalian sEH is encoded by the Ephx2 gene and extensively expressed in multiple organs/tissues including vasculatures; it converts epoxides to diols by adding water to open the epoxide, thus inactivating EETs (Harris and Hammock, 2013). The majority of cardiovascular protective actions elicited by pharmacological inhibition of sEH activity using sEH inhibitors (sEHIs) or genetic deletion of the Ephx2 gene have been ascribed to be due to increases in circulating and tissue/cellular EET levels (Fang et al., 2004; Deng et al., 2011).

Noteworthily, the estrogen-potentiation of EET production takes place primarily in the presence of endothelial dysfunction, whereas estrogen-downregulation of sEH occurs inherently in physiological conditions. The identification of sexual dimorphism of sEH was originally reported around the 1980's, where sEH activity was found to be remarkably higher in organs/tissues of male and OV female mice in comparison to intact females (Denlinger and Vesell, 1989; Pinot et al., 1995), and further validated by a female-specific downregulation of sEH expression (Zhang et al., 2009; Kandhi et al., 2015; Froogh et al., 2016; Qin et al., 2016). This is reminiscent of the phenomenon known as the "male-specific hypotensive response to sEH deficiency," where the deletion of the Ephx2 gene in male mice elicited a significant reduction in blood pressure, with minimal hypotensive effects on female mice (Sinal et al., 2000). We found that $\mathrm{KO}$ of the Ephx2 gene (sEH-KO) or treatment with sEHIs in male mice reduced their blood pressure to the level comparable to that of wild type (WT) females; in the latter, disruption of the Ephx2 gene further reduced blood pressure but with significantly smaller decrement than in male counterparts (Kandhi et al., 2015; Qin et al., 2015a; 2016; Froogh et al., 2016). This dose-dependent-like phenomenon implies that females may heritably possess a mechanism that imitates an action caused by the deletion of the Ephx2 gene in males, making females less sensitive to an additional disruption of the gene. By using in vivo and in vitro models, we demonstrate that estrogen, through ERs, methylates the Ephx2 gene promoter to silence its transcriptional activity, a response that involves multiple transcription factor-driven regulatory signaling (Yang et al., 2018). This study provides mechanistically based explanations for the sexually dimorphic expression of $\mathrm{sEH}$ and all the consequences arising therefrom, that will be discussed in the forthcoming sections.

\section{ROLES OF EETS IN SYSTEMIC CIRCULATION, AS A FUNCTION OF ESTROGEN-DEPENDENT DOWNREGULATION OF SEH}

Female-specific downregulation of sEH expression stabilizes EETs and functionally potentiates EET bioavailability.

In the cerebral circulation, studies using animal models of ischemia demonstrated that estrogen suppression of $\mathrm{sEH}$ was responsible for the female-favorable protection against cerebral ischemic damages in an EET-dependent manner (Fairbanks et al., 2012; Davis et al., 2013).

In the coronary circulation, our laboratories have provided evidence indicating sex-different adaptation of cardiac performance, by conducting experiments on Langendorffperfusion preparations. In physiological conditions, challenged with same increases in preload, female hearts displayed significantly greater cardiac contractility, associated with enhanced coronary blood flow and lower vascular resistance compared to male hearts (Qin et al., 2016). Isolated coronary arteries from female hearts exhibited significantly attenuated pressure-induced myogenic vasoconstriction compared to male arteries, responses that were prevented by 14,15-EEZE (a putative inhibitor of EETs) (Froogh et al., 2016; Qin et al., 2016). These female-specific adaptations were also observed in male $s \mathrm{EH}-\mathrm{KO}$ mice, implying that estrogen downregulation of $\mathrm{sEH}$ duplicates actions of Ephx2 deletion, yielding identical patterns of attenuated coronary myogenic responses, enhanced coronary perfusion and improved cardiac contractility, along with similar cardiac EET metabolic profiles (a great ratio of EETs/DHETs) among female WT, male sEH-KO mice and male WT mice treated with sEHIs (Sun et al., 2014; Qin et al., 2015b). In pathological conditions, Seubert's group using cardiac ischemia models provided strong evidence indicating an EET-driven protection against ischemia/reperfusion-induced cardiac injury in sEH deficient animals (Chaudhary et al., 2009).

In the skeletal muscle and splanchnic circulations, isolated arterioles from female WT mice exhibited significantly greater magnitude of EET-mediated SSID, accompanied with attenuated arteriolar tone than those of male WT controls, responses that were also elicited in vessels isolated from male sEH-KO mice (Sun et al., 2014; Qin et al., 2015a).

Collectively, the evidence of estrogen-dependent suppression of Ephxz/sEH expression provides a novel mechanistic explanation, in addition to the estrogen potentiation of NO-mediated responses (Huang and Kaley, 2004), for the 
better cardiac performance and lower incidence of ischemic cardiovascular diseases in women than men.

\section{ROLES OF ESTROGEN-DRIVEN EET PRODUCTION IN PULMONARY CIRCULATION}

The systemic circulation is benefited from EETs, which then, creates a question as to whether the increase in pulmonary EETs is a "friend or foe?" In addition to the typical feature of low oxygenated blood in the pulmonary artery (PA), there are other two unique features in the pulmonary circulation that differ from the systemic circulation: (1) hypoxia pulmonary vasoconstriction (HPV) (Sylvester et al., 2012) that is reminiscent of hypoxia-induced vasodilation in systemic vasculatures (Busse et al., 1983) and (2) EET-induced pulmonary vasoconstriction via perhaps, depolarizing PA smooth muscle cells (SMC) (Zhu et al., 2000; Kandhi et al., 2016) in contrast to EET-induced vasodilation in systemic vasculatures via hyperpolarizing vascular SMC. Although currently, there is no specific explanation for the divergent responsiveness to hypoxia and EETs in the pulmonary circulation, both features seem to be relevant to estrogens, which therefore, sheds light upon the categorization of pulmonary hypertension $(\mathrm{PH})$ as a disease with female-specific prevalence (Miller, 2012).

Thus, human studies show a female to male ratio of $4.3: 1$ among the total PH patients (Walker et al., 2006), and 4.1:1 in the idiopathic PH (IPAH) subcategory (Badesch et al., 2010). This sexual dimorphism in $\mathrm{PH}$ is evoked at least in part, by estrogen, as evidenced by a high $\mathrm{PH}$ prevalence in women who have taken oral contraceptives (Kleiger et al., 1976), received hormone replacement therapy (Sweeney and Voelkel, 2009) or had enhanced aromatase activity (Roberts et al., 2009). Also, male IPAH patients exist with significantly higher plasma estrogen levels, or a greater ratio of estrogen to testosterone than healthy males (Wu et al., 2018).

Clinical studies provide correlations between the polymorphism of gene(s) involving estrogen metabolisms and the female predisposition to $\mathrm{PH}$ (Austin et al., 2009). In general, the mutation in the BMPR2 gene turns out to be one of the most important genetic-based alterations responsible for the sex-bias in IPAH (Morse et al., 2001; Austin et al., 2013; Dempsie et al., 2011; White et al., 2011), as the penetrance of PH among $B M P R 2$ mutation carriers shows a $42 \%$ penetrance in females vs. $14 \%$ in males (Larkin et al., 2012). Female BMPR2 mutation carriers with $\mathrm{PH}$ exhibit a ten-fold reduction in CYP1B1 gene expression (West et al., 2008; White et al., 2012), followed by altered estrogen metabolism, manifested by a significantly lower ratio of $2-\mathrm{OHE}^{1} / 2$ to $16 \alpha-\mathrm{OHE}_{1}$ (Austin et al., 2009). This estrogen dysmetabolism shifts the balance away from $2-\mathrm{OHE}^{1 / 2}$ induced anti-mitogenic effects toward $16 \alpha-\mathrm{OHE}_{1}$-stimulated pulmonary mitogenic and genotoxic pathways (Austin et al., 2013). Direct binding of $\mathrm{ER} \alpha$ to the promoter of BMPR2 gene silences its expression, which disrupts downstream signaling of bone morphogenetic protein-dependent ligand binding, kinase activation and heteromeric dimer formation etc. (Lane et al., 2000; Austin et al., 2012; Johansen et al., 2016).

To date, there is little attention paid to the intrinsic causation between the estrogen-driven physiological downregulation of Ephx2 gene/sEH and female-susceptibility to be pulmonary hypertensive, a topic that is being investigated in our laboratories. As reported, increases in pulmonary EETs caused by estrogen downregulation of $\mathrm{sEH}$, knockdown of $\mathrm{sEH}$ and treatment with sEHIs propel HPV and promote elevation of PA pressure in response to acute hypoxia (Keseru et al., 2010; Kandhi et al., 2015, 2017). Underlying mechanisms responsible for the EET-dependent potentiation of HPV and hypoxia-induced pulmonary hypertension $(\mathrm{HPH})$ remain elusive; however, roles of vasoconstrictor prostanoids and Rho kinase in this process have emerged, as shown that enhanced hypoxic responses were prevented by 14,15-EEZE, and by inhibition of COXs and Rho kinase, respectively (Keseru et al., 2008; Kandhi et al., 2017). Additionally, the membrane translocation of a TRPC6-V5 fusion protein within PASMC was sensitive to 14,15-EEZE, and hypoxia-induced EET-mediated increases in pulmonary pressure failed to be elicited in mouse lungs that were deficient in TRPC6 (Keseru et al., 2008). Thus, the interaction among estrogen/ERs, sEH/EETs and hypoxia/TRPC6/Rho kinase/PGs works reciprocally, forming a feedback loop in a pattern of cause and result for one another, to elevate PA pressure.

Furthermore, the sex disparity during the development of $\mathrm{HPH}$ was evaluated by using radio-telemetry to dynamically monitor changes in rat PA pressure. Figure 1 shows that under a comparable basal/normoxic PA pressure, male and female rats displayed a time-dependent elevation of PA pressure in response to hypoxia, which, however, occurred earlier accompanied with greater magnitude in females than males, revealing female oversensitivity to hypoxia. This hypoxic responsiveness in female rats was also observed in sEHI-treated male rats, indicating the role of EETs in the event. Noteworthily, during the process of

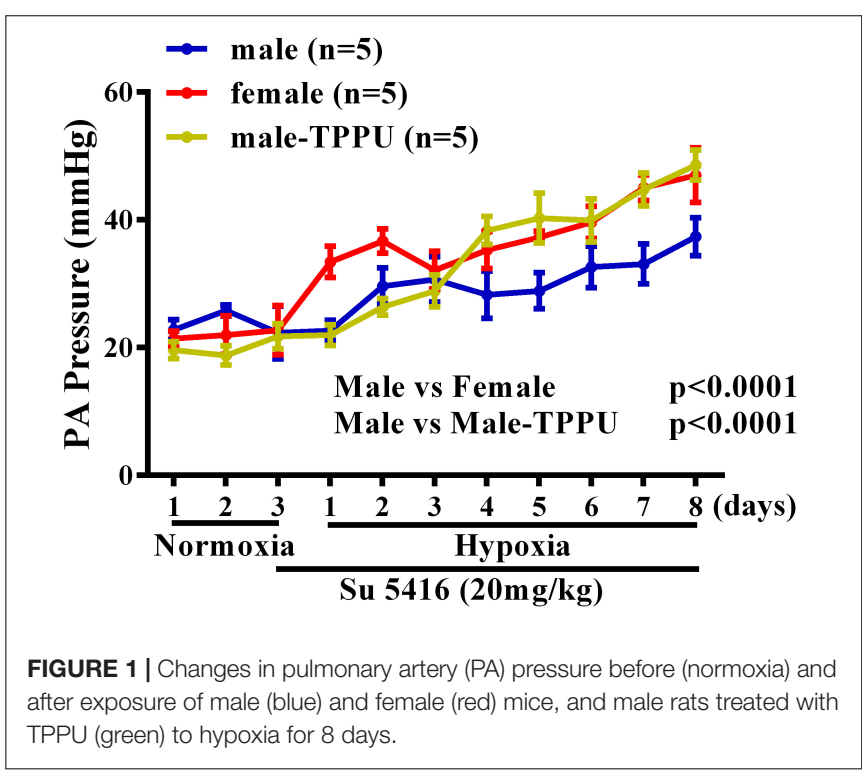


HPH development, hypoxia per se, enables to stimulate EET synthesis (Michaelis et al., 2005) and suppress sEH expression in a sex-independent manner (Petruzzelli et al., 1992), which exacerbate EET-mediated HPV in both genders. Alternatively, when $\mathrm{PH}$ is ultimately established, female patients paradoxically exhibited less impairment in right ventricle (RV) function, indicating better $\mathrm{PH}$ prognosis compared to males (Mair et al., 2014).

A lack of consensus regarding to roles of estrogen in $\mathrm{PH}$ is presumably, due to the presence of the "estrogen paradox" that is characterized by divergent actions (detrimental and beneficial) of estrogens in the incidence and prognosis of PH (Lahm et al., 2014). Figure 2 interprets "estrogen paradox" to mean that Eph $x 2 / \mathrm{sEH}$ and CYP are intimately involved in the pathogenesis of $\mathrm{HPH}$, via being targeted and dysregulated by estrogen and hypoxia to increase pulmonary EET bioavailability. Female PAs are capable of maintaining a normal pressure in response to physiological increases in pulmonary EETs due to the presence of compensatory balancing mechanisms such as estrogenupregulation of eNOS/NO, but bear hyper-responsiveness to acquired pathological challenges such as hypoxia or altered estrogen metabolism, leading to the female susceptibility to $\mathrm{PH}$. Alternatively, all types of $\mathrm{PH}$ regardless of their specific etiologies, undergo/share a common progressive process that involves multiple pathological alterations including but not limited to, the endothelial dysfunction, enhanced oxidative stress and inflammation, vascular remodeling and formation of occlusive lesions, leading to RV hypertrophy and dysfunction, and eventually right heart failure (Rabinovitch, 2012), whereas, all of these pathological alterations can be challenged against by estrogens. For instance, in sugen-hypoxia ( $\mathrm{SuHx}-\mathrm{HP})$-induced $\mathrm{PH}$, estrogen improves RV function via inotropic effects on myocardium (Liu et al., 2014) and restoration of conduit PA compliance (Liu et al., 2015). In monocrotaline (MCT)-induced $\mathrm{PH}$, estrogen prevents MCT-induced impairment of antioxidant capacity to preserve myocardial function (Bal et al., 2013). This points to a non-specific pattern of estrogen-driven improvement of $\mathrm{PH}$ prognosis, which is neither selectively triggered by a specific model of $\mathrm{PH}$, nor does it direct particular target(s), and provides explanations for the better prognosis with higher survival rate in female than male $\mathrm{PH}$ patients.

Currently, clinical trials designed to evaluate the protection of sEHIs if any, for patients with chronic obstructive pulmonary diseases (COPD) reveled that GSK alleviated endothelial dysfunction in COPD patients (Lazaar et al., 2016; Yang et al., 2017). Since HPH, as well as COPD, is associated with downregulation of $\mathrm{sEH}$ and upregulation of EET production, the improvement of endothelial function elicited by GKS in COPD patents may not be purely, mediated by the inhibition of $\mathrm{sEH}$ per se, but rather by alternative pathways. Indeed, in

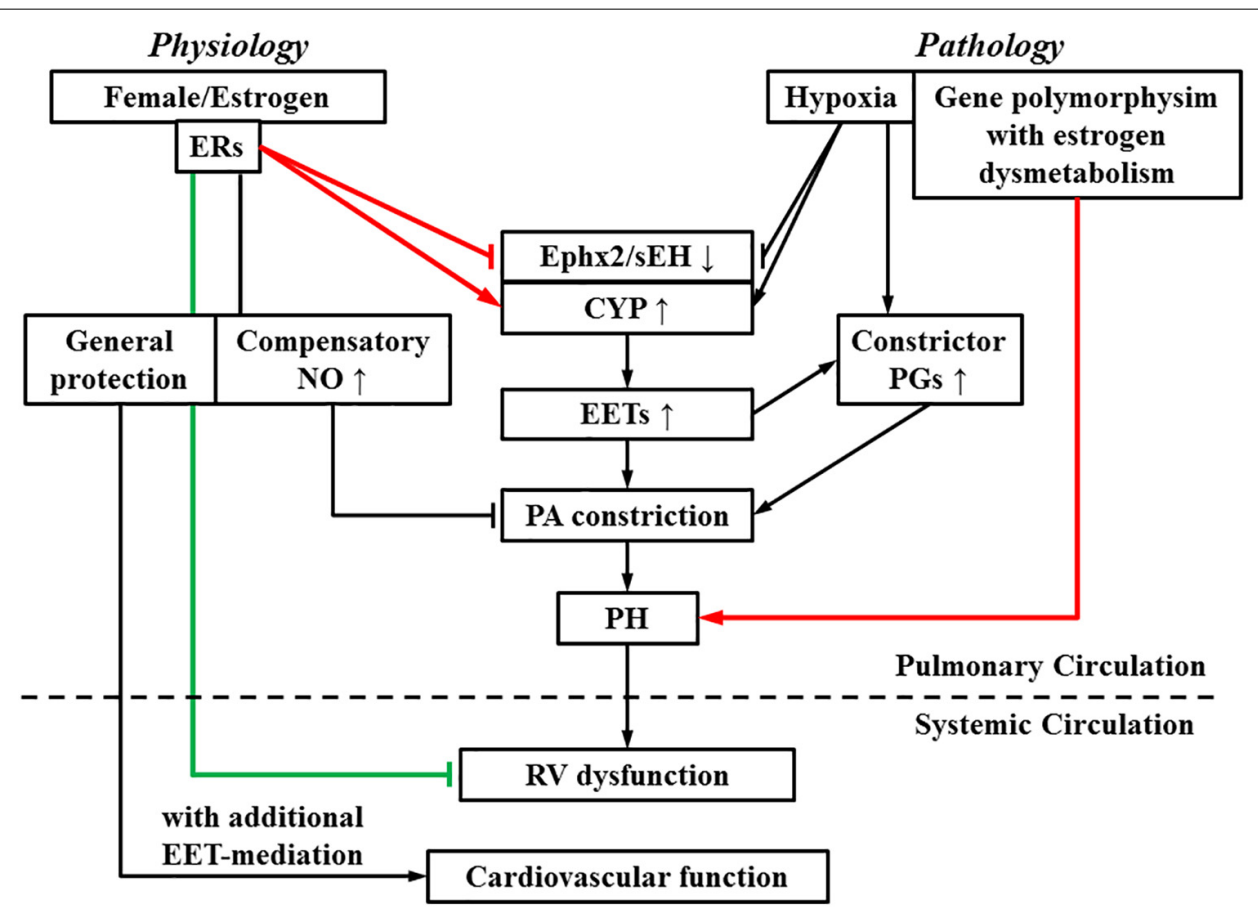

FIGURE 2 | During the process of switch from a physiologically based downregulation of sEH by estrogen/estrogen receptors (ERs) to the pathological development of pulmonary hypertension (PH), multiple pathological stimuli operate in concert, to interrupt the normal physiological balance, tipping the balance toward PA constrictor axis. As indicated, pathological increases in EETs, as a function of upregulation of EET syntheses (CYP) or suppression of Ephx2/sEH elicit PA constriction and promote actions of constrictor prostaglandins (PGs), resulting in an EET-potentiation of PH, which eventually impairs right ventricular (RV) function. On the other hand, RV dysfunction is alleviated by estrogen. The systemic circulation is also benefited by estrogen- and EET-mediated protections. Arrow-head indicates promotion. Flat-head indicates suppression. Estrogen-promotion of incidence and -improvement of prognosis of $\mathrm{PH}$ are demarcated by lines designated in red and green respectively. 
addition to targeting sEH, sEHIs are capable of binding with other enzymes due to the presence of multi-target ligands. For instance, PTUPB is a tight COX-2 binder (Hwang et al., 2018) and TPPU selectively inhibits p38 $\beta$ kinase to block downstreamlocated NF-кB-dependent signaling (Liang et al., 2018). As such, pharmacological inhibition of sEH to stabilize EETs may instigate PA vasoconstriction but somehow, mitigate pathological progression in the pulmonary circulation.

\section{SUMMARY AND PERSPECTIVES}

We briefly summarized the pathophysiological significance of potentiating EET production and/or inhibiting EET hydrolysis, as a function of estrogen, in the regulation of systemic and pulmonary circulations. In the systemic circulation, increases in EETs afford better cardiovascular performance and lower incidence of ischemic diseases in women. In the pulmonary circulation, clinical development of $\mathrm{PH}$ appears to require "two hits" that can be triggered by either genetics (sex and

\section{REFERENCES}

Archer, S. L., Gragasin, F. S., Wu, X., Wang, S., McMurtry, S., Kim, D. H., et al. (2003). Endothelium-derived hyperpolarizing factor in human internal mammary artery is 11,12-epoxyeicosatrienoic acid and causes relaxation by activating smooth muscle BK(Ca) channels. Circulation 107, 769-776. doi: 10. 1161/01.CIR.0000047278.28407.C2

Austin, E. D., Cogan, J. D., West, J. D., Hedges, L. K., Hamid, R., Dawson, E. P., et al. (2009). Alterations in oestrogen metabolism: implications for higher penetrance of familial pulmonary arterial hypertension in females. Eur. Respir. J. 34, 1093-1099. doi: 10.1183/09031936.00010409

Austin, E. D., Hamid, R., Hemnes, A. R., Loyd, J. E., Blackwell, T., Yu, C., et al. (2012). BMPR2 expression is suppressed by signaling through the estrogen receptor. Biol. Sex Differ. 3:6. doi: 10.1186/2042-6410-3-6

Austin, E. D., Lahm, T., West, J., Tofovic, S. P., Johansen, A. K., MacLean, M. R., et al. (2013). Gender, sex hormones and pulmonary hypertension. Pulm. Circ. 3, 294-314. doi: 10.4103/2045-8932.114756

Badesch, D. B., Raskob, G. E., Elliott, C. G., Krichman, A. M., Farber, H. W., Frost, A. E., et al. (2010). Pulmonary arterial hypertension: baseline characteristics from the REVEAL Registry. Chest 137, 376-387. doi: 10.1378/chest.09-1140

Bal, E., Ilgin, S., Atli, O., Ergun, B., and Sirmagul, B. (2013). The effects of gender difference on monocrotaline-induced pulmonary hypertension in rats. Hum. Exp. Toxicol. 32, 766-774. doi: 10.1177/096032711347 7874

Brandes, R. P., Schmitz-Winnenthal, F. H., Feletou, M., Godecke, A., Huang, P. L., Vanhoutte, P. M., et al. (2000). An endothelium-derived hyperpolarizing factor distinct from NO and prostacyclin is a major endothelium-dependent vasodilator in resistance vessels of wild-type and endothelial NO synthase knockout mice. Proc. Natl. Acad. Sci. U.S.A. 97, 9747-9752. doi: 10.1073/pnas. 97.17.9747

Busse, R., Pohl, U., Kellner, C., and Klemm, U. (1983). Endothelial cells are involved in the vasodilatory response to hypoxia. Pflugers Arch. 397, 78-80. doi: 10.1007/BF00585175

Chaudhary, K. R., Batchu, S. N., and Seubert, J. M. (2009). Cytochrome P450 enzymes and the heart. IUBMB Life 61, 954-960. doi: 10.1002/iub.241

Davis, C. M., Fairbanks, S. L., and Alkayed, N. J. (2013). Mechanism of the sex difference in endothelial dysfunction after stroke. Transl. Stroke Res. 4:381-389. doi: 10.1007/s12975-012-0227-0

Dempsie, Y., Nilsen, M., White, K., Mair, K. M., Loughlin, L., Ambartsumian, N., et al. (2011). Development of pulmonary arterial hypertension in mice overexpressing S100A4/Mts1 is specific to females. Respir. Res. 12, 159. doi: 10.1186/ 1465-9921-12-159 gene polymorphisms), environmental factors (hypoxia and sex hormone dysmetabolism), or both (female with hypoxia). Thus, from a pros and cons point of view, targeting CYP and sEH may prove to be a double-edged sword with beneficial and adverse effects on systemic and pulmonary circulatory systems; this brings concerns surrounding the use of sEHIs as therapeutic regimens, or the consideration of sEHI-related clinical trials in female populations who bear hyper-responsiveness to acquired pathological insults (as second hits) to the respiratory system.

\section{AUTHOR CONTRIBUTIONS}

$\mathrm{AH}$ and DS contributed equally to the literature search, figures, and writing, and gave their final approval of the manuscript.

\section{FUNDING}

This work was supported by grants HL070653 and HL129797.

Deng, Y., Edin, M. L., Theken, K. N., Schuck, R. N., Flake, G. P., Kannon, M. A., et al. (2011). Endothelial CYP epoxygenase overexpression and soluble epoxide hydrolase disruption attenuate acute vascular inflammatory responses in mice. FASEB J. 25, 703-713. doi: 10.1096/fj.10-171488

Denlinger, C. L., and Vesell, E. S. (1989). Hormonal regulation of the developmental pattern of epoxide hydrolases. Studies in rat liver. Biochem. Pharmacol. 38, 603-610. doi: 10.1016/0006-2952(89)90205-0

Fairbanks, S. L., Young, J. M., Nelson, J. W., Davis, C. M., Koerner, I. P., and Alkayed, N. J. (2012). Mechanism of the sex difference in neuronal ischemic cell death. Neuroscience 219, 183-191. doi: 10.1016/j.neuroscience.2012.05.048

Fang, X., Weintraub, N. L., McCaw, R. B., Hu, S., Harmon, S. D., Rice, J. B., et al. (2004). Effect of soluble epoxide hydrolase inhibition on epoxyeicosatrienoic acid metabolism in human blood vessels. Am. J. Physiol. Heart Circ. Physiol. 287, H2412-H2420. doi: 10.1152/ajpheart.00527.2004

Fleming, I. (2004). Cytochrome P450 epoxygenases as EDHF synthase(s). Pharmacol. Res. 49, 525-533. doi: 10.1016/j.phrs.2003.11.016

Froogh, G., Qin, J., Kandhi, S., Le, Y., Jiang, H., Luo, M., et al. (2016). Female-favorable attenuation of coronary myogenic constriction via reciprocal activations of epoxyeicosatrienoic acids and nitric oxide. Am. J. Physiol. Heart Circ. Physiol. 310, H1448-H1454. doi: 10.1152/ajpheart.00906.2015

Galipeau, D., Verma, S., and McNeill, J. H. (2002). Female rats are protected against fructose-induced changes in metabolism and blood pressure. Am. J. Physio.l Heart Circ. Physiol. 283, H2478-H2484. doi: 10.1152/ajpheart.00243. 2002

Harris, T. R., and Hammock, B. D. (2013). Soluble epoxide hydrolase: gene structure, expression and deletion. Gene 526, 61-74. doi: 10.1016/j.gene.2013. 05.008

Huang, A., and Kaley, G. (2004). Gender-specific regulation of cardiovascular function: estrogen as key player. Microcirculation 11, 9-38. doi: 10.1080/ 10739680490266162

Huang, A., Sun, D., Carroll, M. A., Jiang, H., Smith, C. J., Connetta, J. A., et al. (2001a). EDHF mediates flow-induced dilation in skeletal muscle arterioles of female eNOS-KO mice. Am. J. Physiol. Heart Circ. Physiol. 280, H2462-H2469.

Huang, A., Wu, Y., Sun, D., Koller, A., and Kaley, G. (2001b). Effect of estrogen on flow-induced dilation in NO deficiency: role of prostaglandins and EDHF. J. Appl. Physiol. 91, 2561-2566.

Huang, A., Sun, D., Jacobson, A., Carroll, M. A., Falck, J. R., and Kaley, G. (2005). Epoxyeicosatrienoic acids are released to mediate shear stress-dependent hyperpolarization of arteriolar smooth muscle. Circ. Res. 96, 376-383. doi: 10.1161/01.RES.0000155332.17783.26

Huang, A., Sun, D., Wu, Z., Yan, C., Carroll, M. A., Jiang, H., et al. (2004). Estrogen elicits cytochrome P450-mediated flow-induced dilation of arterioles in NO 
deficiency: role of PI3K-Akt phosphorylation in genomic regulation. Circ. Res. 94, 245-252. doi: 10.1161/01.RES.0000111525.96232.46

Hwang, S. H., Gaine, S., Morisseau, C., Yang, J., Wagner, K., Gilson, M. K., et al. (2018). Dual inhibitors of cyclooxygenase-2 and soluble epoxie hydrolase: studies of binding modes $t$ the active sites and time-dependency of inhibition and development of water-soluble prodrug. FASEB J. 32.

Imig, J. D. (2012). Epoxides and soluble epoxide hydrolase in cardiovascular physiology. Physiol. Rev. 92, 101-130. doi: 10.1152/physrev.00021. 2011

Johansen, A. K., Dean, A., Morecroft, I., Hood, K., Nilsen, M., Loughlin, L., et al. (2016). The serotonin transporter promotes a pathological estrogen metabolic pathway in pulmonary hypertension via cytochrome P450 1B1. Pulm. Circ. 6:82-92. doi: 10.1086/685023

Kandhi, S., Froogh, G., Qin, J., Luo, M., Wolin, M. S., Huang, A., et al. (2016). EETs Elicit Direct Increases in Pulmonary Arterial Pressure in Mice. Am. J. Hypertens. 29, 598-604. doi: 10.1093/ajh/hpv148

Kandhi, S., Qin, J., Froogh, G., Jiang, H., Luo, M., Wolin, M. S., et al. (2015). EETdependent potentiation of pulmonary arterial pressure: sex-different regulation of soluble epoxide hydrolase. Am. J. Physiol. Lung Cell. Mol. Physiol. 309, L1478-L1486. doi: 10.1152/ajplung.00208.2015

Kandhi, S., Zhang, B., Froogh, G., Qin, J., Alruwaili, N., Le, Y., et al. (2017). EETs promote hypoxic pulmonary vasoconstriction via constrictor prostanoids. Am. J. Physiol. Lung Cell. Mol. Physiol. 313, L350-L359. doi: 10.1152/ajplung.00038. 2017

Keseru, B., Barbosa-Sicard, E., Popp, R., Fisslthaler, B., Dietrich, A., Gudermann, T., et al. (2008). Epoxyeicosatrienoic acids and the soluble epoxide hydrolase are determinants of pulmonary artery pressure and the acute hypoxic pulmonary vasoconstrictor response. FASEB J. 22, 4306-4315. doi: 10.1096/fj.08-112821

Keseru, B., Barbosa-Sicard, E., Schermuly, R. T., Tanaka, H., Hammock, B. D., Weissmann, N., et al. (2010). Hypoxia-induced pulmonary hypertension: comparison of soluble epoxide hydrolase deletion vs. inhibition. Cardiovasc. Res. 85, 232-240. doi: 10.1093/cvr/cvp281

Kleiger, R. E., Boxer, M., Ingham, R. E., and Harrison, D. C. (1976). Pulmonary hypertension in patients using oral contraceptives. A report of six cases. Chest 69, 143-147. doi: 10.1378/chest.69.2.143

Lahm, T., Tuder, R. M., and Petrache, I. (2014). Progress in solving the sex hormone paradox in pulmonary hypertension. Am. J. Physiol. Lung Cell. Mol. Physiol. 307, L7-L26. doi: 10.1152/ajplung.00337.2013

Lane, K. B., Machado, R. D., Pauciulo, M. W., Thomson, J. R., Phillips, J. A., Loyd, J. E., et al. (2000). Heterozygous germline mutations in BMPR2, encoding a TGF-beta receptor, cause familial primary pulmonary hypertension. Nat. Genet. 26, 81-84. doi: $10.1038 / 79226$

Larkin, E. K., Newman, J. H., Austin, E. D., Hemnes, A. R., Wheeler, L., Robbins, I. M., et al. (2012). Longitudinal analysis casts doubt on the presence of genetic anticipation in heritable pulmonary arterial hypertension. Am. J. Respir. Crit. Care Med. 186, 892-896. doi: 10.1164/rccm.201205-0886OC

Lazaar, A. L., Yang, L., Boardley, R. L., Goyal, N. S., Robertson, J., Baldwin, S. J., et al. (2016). Pharmacokinetics, pharmacodynamics and adverse event profile of GSK2256294, a novel soluble epoxide hydrolase inhibitor. Br. J. Clin. Pharmacol. 81, 971-979. doi: 10.1111/bcp.12855

Lee, J., Dahl, M., Grande, P., Tybjaerg-Hansen, A., and Nordestgaard, B. G. (2010). Genetically reduced soluble epoxide hydrolase activity and risk of stroke and other cardiovascular disease. Stroke 41, 27-33. doi: 10.1161/STROKEAHA.109. 567768

Liang, Z., Morisseau, C., Hwang, S. H., Hammock, B. D., Li, Q. X. (2018). A dual-inhibitor of soluble epoxide hydrolase and p3 kinase alleviating Tau hyperphorylation and amyloid neurotoxicity for potential treatment of neuroinflammation in Alzheimer's disease. FASEB J. 32.

Liu, A., Schreier, D., Tian, L., Eickhoff, J. C., Wang, Z., Hacker, T. A., et al. (2014). Direct and indirect protection of right ventricular function by estrogen in an experimental model of pulmonary arterial hypertension. Am. J. Physiol. Heart Circ. Physiol. 307, H273-H283. doi: 10.1152/ajpheart.00758. 2013

Liu, A., Tian, L., Golob, M., Eickhoff, J. C., Boston, M., and Chesler, N. C. (2015). 17beta-Estradiol Attenuates Conduit Pulmonary Artery Mechanical Property Changes With Pulmonary Arterial Hypertension. Hypertension 66, 1082-1088. doi: 10.1161/HYPERTENSIONAHA.115.05843
Ma, B., Xiong, X., Chen, C., Li, H., Xu, X., Li, X., et al. (2013). Cardiacspecific overexpression of CYP2J2 attenuates diabetic cardiomyopathy in male streptozotocin-induced diabetic mice. Endocrinology 154, 2843-2856. doi: 10. 1210/en.2012-2166

Mair, K. M., Johansen, A. K., Wright, A. F., Wallace, E., and MacLean, M. R. (2014). Pulmonary arterial hypertension: basis of sex differences in incidence and treatment response. Br. J. Pharmacol. 171, 567-579. doi: 10.1111/bph. 12281

Michaelis, U. R., Fisslthaler, B., Barbosa-Sicard, E., Falck, J. R., Fleming, I., and Busse, R. (2005). Cytochrome P450 epoxygenases 2C8 and 2C9 are implicated in hypoxia-induced endothelial cell migration and angiogenesis. J. Cell. Sci. 118, 5489-5498. doi: $10.1242 /$ jcs.02674

Miller, V. M. (2012). In pursuit of scientific excellence: sex matters. Am. J. Physiol. Heart Circ. Physiol. 302, H1771-H1772. doi: 10.1152/ajpheart.00073.2012

Morse, J. H., Deng, Z., and Knowles, J. A. (2001). Genetic aspects of pulmonary arterial hypertension. Ann. Med. 33, 596-603. doi: 10.3109/0785389010900 2105

Newman, J. W., Morisseau, C., and Hammock, B. D. (2005). Epoxide hydrolases: their roles and interactions with lipid metabolism. Prog. Lipid Res. 44, 1-51. doi: 10.1016/j.plipres.2004.10.001

Petruzzelli, S., Franchi, M., Gronchi, L., Janni, A., Oesch, F., Pacifici, G. M., et al. (1992). Cigarette smoke inhibits cytosolic but not microsomal epoxide hydrolase of human lung. Hum. Exp. Toxicol. 11, 99-103. doi: 10.1177/ 096032719201100207

Pinot, F., Grant, D. F., Spearow, J. L., Parker, A. G., and Hammock, B. D. (1995). Differential regulation of soluble epoxide hydrolase by clofibrate and sexual hormones in the liver and kidneys of mice. Biochem. Pharmacol. 50, 501-508. doi: 10.1016/0006-2952(95)00167-X

Qin, J., Kandhi, S., Froogh, G., Jiang, H., Luo, M., Sun, D., et al. (2015a). Sexually dimorphic phenotype of arteriolar responsiveness to shear stress in soluble epoxide hydrolase-knockout mice. Am. J. Physiol. Heart Circ. Physiol. 309, H1860-H1866. doi: 10.1152/ajpheart.00568.2015

Qin, J., Sun, D., Jiang, H., Kandhi, S., Froogh, G., Hwang, S. H., et al. (2015b). Inhibition of soluble epoxide hydrolase increases coronary perfusion in mice. Physiol. Rep. 3:e12427. doi: 10.14814/phy2.12427

Qin, J., Le, Y., Froogh, G., Kandhi, S., Jiang, H., Luo, M., et al. (2016). Sexually dimorphic adaptation of cardiac function: roles of epoxyeicosatrienoic acid and peroxisome proliferator-activated receptors. Physiol. Rep. 4:e12838. doi: 10.14814 /phy2.12838

Rabinovitch, M. (2012). Molecular pathogenesis of pulmonary arterial hypertension. J. Clin. Invest. 122, 4306-4313. doi: 10.1172/JCI60658

Roberts, K. E., Fallon, M. B., Krowka, M. J., Brown, R. S., Trotter, J. F., Peter, I., et al. (2009). Genetic risk factors for portopulmonary hypertension in patients with advanced liver disease. Am. J. Respir. Crit. Care Med. 179, 835-842. doi: $10.1164 / \mathrm{rccm} .200809-1472 \mathrm{OC}$

Roman, R. J. (2002). P-450 metabolites of arachidonic acid in the control of cardiovascular function. Physiol. Rev. 82, 131-185. doi: 10.1152/physrev.00021. 2001

Scotland, R. S., Madhani, M., Chauhan, S., Moncada, S., Andresen, J., Nilsson, H., et al. (2005). Investigation of vascular responses in endothelial nitric oxide synthase/cyclooxygenase-1 double-knockout mice: key role for endothelium-derived hyperpolarizing factor in the regulation of blood pressure in vivo. Circulation 111, 796-803. doi: 10.1161/01.CIR.0000155238. 70797.4E

Sinal, C. J., Miyata, M., Tohkin, M., Nagata, K., Bend, J. R., and Gonzalez, F. J. (2000). Targeted disruption of soluble epoxide hydrolase reveals a role in blood pressure regulation. J. Biol. Chem. 275, 40504-40510. doi: 10.1074/jbc. M008106200

Song, D., Arikawa, E., Galipeau, D. M., Yeh, J. N., Battell, M. L., Yuen, V. G., et al. (2005). Chronic estrogen treatment modifies insulin-induced insulin resistance and hypertension in ovariectomized rats. Am. J. Hypertens. 18, 1189-1194. doi: 10.1016/j.amjhyper.2005.04.003

Sun, D., Cuevas, A. J., Gotlinger, K., Hwang, S. H., Hammock, B. D., Schwartzman, M. L., et al. (2014). Soluble epoxide hydrolase-dependent regulation of myogenic response and blood pressure. Am. J. Physiol. Heart Circ. Physiol. 306, H1146-H1153. doi: 10.1152/ajpheart.00920.2013

Sun, D., Huang, A., Smith, C. J., Stackpole, C. J., Connetta, J. A., Shesely, E. G., et al. (1999). Enhanced release of prostaglandins contributes to flow-induced 
arteriolar dilation in eNOS knockout mice. Circ. Res. 85, 288-293. doi: 10.1161/ 01.RES.85.3.288

Sun, D., Jiang, H., Wu, H., Yang, Y., Kaley, G., and Huang, A. (2011). A novel vascular EET synthase: role of CYP2C7. Am. J. Physiol. Regul. Integr. Comp. Physiol. 301, R1723-R1730. doi: 10.1152/ajpregu.00382.2011

Sun, D., Liu, H., Yan, C., Jacobson, A., Ojaimi, C., Huang, A., et al. (2006). COX2 contributes to the maintenance of flow-induced dilation in arterioles of eNOS-knockout mice. Am. J. Physiol. Heart Circ. Physiol. 291, H1429-H1435. doi: 10.1152/ajpheart.01130.2005

Sun, D., Yang, Y. M., Jiang, H., Wu, H., Ojaimi, C., Kaley, G., et al. (2010). Roles of CYP2C29 and RXR gamma in vascular EET synthesis of female mice. Am. J. Physiol. Regul. Integr. Comp. Physiol. 298, R862-R869. doi: 10.1152/ajpregu. 00575.2009

Sweeney, L., and Voelkel, N. F. (2009). Estrogen exposure, obesity and thyroid disease in women with severe pulmonary hypertension. Eur. J. Med. Res. 14, 433-442. doi: 10.1186/2047-783X-14-10-433

Sylvester, J. T., Shimoda, L. A., Aaronson, P. I., and Ward, J. P. (2012). Hypoxic pulmonary vasoconstriction. Physiol. Rev. 92, 367-520. doi: 10.1152/physrev. 00041.2010

Vasudevan, H., Xiang, H., and McNeill, J. H. (2005). Differential regulation of insulin resistance and hypertension by sex hormones in fructose-fed male rats. Am. J. Physiol. Heart Circ. Physiol. 289, H1335-H1342. doi: 10.1152/ajpheart. 00399.2005

Walker, A. M., Langleben, D., Korelitz, J. J., Rich, S., Rubin, L. J., Strom, B. L., et al. (2006). Temporal trends and drug exposures in pulmonary hypertension: an American experience. Am. Heart J. 152, 521-526. doi: 10.1016/j.ahj.2006. 02.020

West, J., Cogan, J., Geraci, M., Robinson, L., Newman, J., Phillips, J. A., et al. (2008). Gene expression in BMPR2 mutation carriers with and without evidence of pulmonary arterial hypertension suggests pathways relevant to disease penetrance. BMC Med. Genomics 1:45. doi: 10.1186/1755-8794-1-45

White, K., Johansen, A. K., Nilsen, M., Ciuclan, L., Wallace, E., Paton, L., et al. (2012). Activity of the estrogen-metabolizing enzyme cytochrome P450 1B1 influences the development of pulmonary arterial hypertension. Circulation 126, 1087-1098. doi: 10.1161/CIRCULATIONAHA.111.062927

White, K., Loughlin, L., Maqbool, Z., Nilsen, M., McClure, J., Dempsie, Y., et al. (2011). Serotonin transporter, sex, and hypoxia: microarray analysis in the pulmonary arteries of mice identifies genes with relevance to human
PAH. Physiol. Genomics 43, 417-437. doi: 10.1152/physiolgenomics.00249. 2010

Wu, W. H., Yuan, P., Zhang, S. J., Jiang, X., Wu, C., Li, Y., et al. (2018). Impact of Pituitary-Gonadal Axis Hormones on Pulmonary Arterial Hypertension in Men. Hypertension 72, 151-158. doi: 10.1161/HYPERTENSIONAHA.118. 10963

Wu, Y., Huang, A., Sun, D., Falck, J. R., Koller, A., and Kaley, G. (2001). Gender-specific compensation for the lack of NO in the mediation of flow-induced arteriolar dilation. Am. J. Physiol. Heart Circ. Physiol. 280, H2456-H2461. doi: 10.1152/ajpheart.2001.280.6. $\mathrm{H} 2456$

Yang, L., Cheriyan, J., Gutterman, D. D., Mayer, R. J., Ament, Z., Griffin, J. L., et al. (2017). Mechanisms of Vascular Dysfunction in COPD and Effects of a Novel Soluble Epoxide Hydrolase Inhibitor in Smokers. Chest 151, 555-563. doi: 10.1016/j.chest.2016.10.058

Yang, Y. M., Sun, D., Kandhi, S., Froogh, G., Zhuge, J., Huang, W., et al. (2018) Estrogen-dependent epigenetic regulation of soluble epoxide hydrolase via DNA methylation. Proc. Natl. Acad. Sci. U.S.A. 115, 613-618. doi: 10.1073/pnas. 1716016115

Zhang, W., Iliff, J. J., Campbell, C. J., Wang, R. K., Hurn, P. D., and Alkayed, N. J. (2009). Role of soluble epoxide hydrolase in the sex-specific vascular response to cerebral ischemia. J. Cereb. Blood Flow Metab. 29, 1475-1481. doi: $10.1038 / \mathrm{jcbfm} .2009 .65$

Zhu, D., Bousamra, M., Zeldin, D. C., Falck, J. R., Townsley, M., Harder, D. R., et al. (2000). Epoxyeicosatrienoic acids constrict isolated pressurized rabbit pulmonary arteries. Am. J. Physiol. Lung Cell. Mol. Physiol. 278, L335-L343. doi: 10.1152/ajplung.2000.278.2.L335

Conflict of Interest Statement: The authors declare that the research was conducted in the absence of any commercial or financial relationships that could be construed as a potential conflict of interest.

Copyright (c) 2018 Huang and Sun. This is an open-access article distributed under the terms of the Creative Commons Attribution License (CC BY). The use, distribution or reproduction in other forums is permitted, provided the original author(s) and the copyright owner(s) are credited and that the original publication in this journal is cited, in accordance with accepted academic practice. No use, distribution or reproduction is permitted which does not comply with these terms. 